\title{
Intracellular sterol transport in eukaryotes, a connection to mitochondrial function?
}

\author{
Roger Schneiter* \\ Department of Medicine, Division of Biochemistry, University of Fribourg, Chemin du Musée 5, CH-1700 Fribourg, Switzerland
}

\begin{abstract}
Eukaryotic cells synthesize sterols in the endoplasmatic reticulum (ER) from where it needs to be efficiently transported to the plasma membrane, which harbors $\sim 90 \%$ of the free sterol pool of the cell. Sterols that are being taken up from the environment, on the other hand, are transported back from the plasma membrane to the ER, where the free sterols are esterified to steryl esters. The molecular mechanisms that govern this bidirectional movement of sterols between the ER and the plasma membrane of eukaryotic cells are only poorly understood. Proper control of this transport is important for normal cell function and development as indicated by fatal human pathologies such as Niemann Pick type $\mathrm{C}$ disease and atherosclerosis, which are characterized by an over-accumulation of free sterols within endosomal membranes and the ER, respectively. Recently, a number of complementary approaches using Saccharomyces cerevisiae as a model organism lead to a more precise characterization of the pathways that control the subcellular transport of sterols and led to the identification of components that directly or indirectly affect sterol uptake at the plasma membrane and its transport back to the ER. A genetic approach that is based on the fact that yeast is a facultative anaerobic organism, which becomes auxotrophic for sterols in the absence of oxygen, resulted in the identification of 17 genes that are required for efficient uptake and/or transport of sterols. Unexpectedly, many of these genes are required for mitochondrial functions. A possible connection between mitochondrial biogenesis and sterol biosynthesis and uptake will be discussed in light of the fact that cholesterol transport into the inner membranes of mitochondria is a well established sterol transport route in vertebrates, where it is required to convert cholesterol into pregnenolone, the precursor of steroids.
\end{abstract}

Keywords: Cholesterol; Lipid transport; Mitochondria; Anaerobiosis; Saccharomyces cerevisiae

\section{Introduction}

Sterols are a major lipid component of the plasma membrane of eukaryotic cells, where they have been proposed to maintain a vital electrochemical gradient across the plasma membrane [1]. In addition, sterols determine other important membrane characteristics that appear to be more transient and are required for polar sorting events during vesicle transport in animal, plant, and fungal cells.

Abbreviations: ER, endoplasmatic reticulum; LDL, low-density lipoprotein; SREBPs, sterol regulatory element binding proteins.

* Tel.: +41 26300 8654; fax: +41 263009735.

E-mail address: roger.schneiter@unifr.ch
Due to the importance of cholesterol in various cell functions, the levels and intracellular distribution of this lipid must be tightly controlled $[2,3]$. While the mechanism that coordinates the de novo synthesis and uptake of cholesterol can now be studied at the molecular level, much less is known about how the relative distribution of cholesterol between the various intracellular membranes is controlled [4]. Proper intracellular transport of cholesterol is essential as indicated by the phenotype of human diseases that have been associated with sterol transport defects. Most notably Niemann-Pick type C1 disease, a fatal neurodegenerative disorder that is characterized by the accumulation of free cholesterol within a late endosomal compartment, or the atherosclerotic macrophages, where an excess of free sterol in the ER leads to the induction of apoptosis [5]. 
Cholesterol is synthesized through a cascade of enzymatic reactions that are located in the ER and the mature free cholesterol is subsequently transported to the plasma membrane, which harbors $\sim 90 \%$ of the free sterol pool of the cell. This transport process may involve both vesicular and non-vesicular components, as indicated by the observation that delivery of newly synthesized cholesterol to the plasma membrane is ATP-dependent, but only partially sensitive to disruption of vesicular transport by brefeldin A [2,3].

Exogenous cholesterol is taken up through receptor-mediated endocytosis of low-density lipoproteins (LDL). Once delivered to late endosome or lysosome, LDL-derived cholesteryl esters are hydrolyzed, and free cholesterol is rapidly cycled back to the plasma membrane and/or the ER for reesterification. Maintenance of the cycle between free and esterified sterols thus relies on a bidirectional transport of sterols between the ER and the plasma membrane and/or an endocytic compartment. This movement of cholesterol from the plasma membrane to the ER is inhibited by hydrophobic amines, progesterone, disruption of the cytoskeleton or that of the acidic compartments, but not by ATP depletion, indicating that it occurs through a non-vesicular route $[2,3]$.

\section{Sterol uptake and transport pathways in yeast}

Yeast is a valuable genetic model organism to characterize basic cellular processes that are conserved in all eukaryotic cells. S. cerevisiae has been established as a model organism to study sterol function and biosynthesis by the pioneering work of Parks and colleagues [6]. The basic steps of sterol synthesis in yeast are the same as in higher eukaryotic cells, even though this fungus does not synthesize cholesterol, but a close structural relative, termed ergosterol [7]. As in mammalian cells, ergosterol is synthesized in the ER, from where it is transported by a poorly characterized mechanism to the plasma membrane, the major ergosterol containing membrane in yeast [8]. Recent characterization of the nature of this forward transport pathway has revealed that the newly synthesized ergosterol equilibrates with a half-time of $10-15 \mathrm{~min}$ with the plasma membrane-localized sterol pool, which corresponds to the transport of approximately $10^{5}$ ergosterol molecules into and out of the plasma membrane per second [9]. This equilibration is independent of the secretory pathway, but delayed in mutant cells that have reduced levels of sphingolipids [10]. This observation was taken to suggest that the "chemical activity" or concentration of free sterol in the ER is the same as that of the plasma membrane, but that the bulk of the plasma membrane sterol is trapped into condensed complexes with sphingolipids, i.e. within lipid microdomains/ "rafts", and thus cannot contribute to the equilibration process between the two membranes, a concept originally formulated by McConnell and colleagues [11]. In such a model, transport of sterols between the two compartments may be facilitated by soluble lipid carrier proteins and/or close apposition of the organellar membranes. The proteins that mediate this sterol transport or those that maintain membrane apposition, however, remain to be identified. Recent structural analysis of a member of the oxysterol-binding protein homologues (Osh proteins), a family of seven proteins in yeast, all of which contain a putative sterol-binding domain, suggests a possible sterol transport cycle in which Osh proteins could act to facilitate the equilibration of sterols between different intracellular membranes [12]. Osh4 binds a single sterol molecule with a $K_{\mathrm{d}}$ of $300 \mathrm{nM}$ within a hydrophobic tunnel that is covered upon sterol binding by a flexible lid domain [12]. A role of Osh proteins in sterol transport or the regulation of sterol distribution is furthermore indicated by the observation that the intracellular sterol distribution is altered in mutant cells that lack Osh function [13].

Examination of a requirement of these Osh proteins for sterol equilibration from the ER to the plasma membrane, however, revealed only a moderate, five-fold delay in mutants lacking Osh function, indicating that Osh proteins may not be sterol transporters by themselves, but that they affect sterol transport indirectly, for example by affecting the ability of the plasma membrane to sequester sterols [9]. The transport of sterols back from the plasma membrane to the ER, however, requires Osh function and is greatly reduced in mutants that fail to synthesize certain phosphoinositide phosphates (PIPs), indicating that PIPs modulate sterol transfer by the Osh proteins, which is consistent with the presence of a PIP-binding pleckstrin homology domain in a subset of the Osh proteins [14]. Whether the Osh proteins indeed act to transport sterols between different membranes or whether they rather function as lipid sensors to regulate lipid transport, however, remains to be established. This discrimination is not a trivial task as exemplified by another well characterized but still poorly understood lipid binding protein, Sec14 and its homologues [15]. Sec14 has originally been identified as a lipid transfer protein that exchanges phosphatidylinositol and phosphatidylcholine. Subsequent genetic analysis, however, revealed that Sec14 is required to repress the synthesis of phosphatidylcholine in the Golgi apparatus and that phosphatidylinositol-binding is dispensable for its essential function to promote vesicle budding from the Golgi complex in vivo [16]. In mammals, one of the oxysterol-binding proteins acts as cholesterol-binding scaffolding protein coordinating the activity of two phosphatases to control the extracellular signal-regulated kinase (ERK) pathway [17]. Remarkably, membrane association of two of the yeast Osh proteins, Osh6 and Osh7, is regulated by Vps4, a member of an ATPase family of proteins implicated in the disassembly of protein complexes, suggesting that this class of ATPases may break the interaction of Osh proteins with their membrane receptors to regulate lipid transport and/or sensing [18].

An excess of free sterol in the ER is buffered by its acylation with long chain fatty acids, which converts the free sterol into a steryl ester. Depending on the growth phase, these steryl esters can reach similar levels as free sterols [19]. In contrast to free sterols, steryl esters are not membrane forming lipids but storage lipids that are packed away into intracellular lipid particles. The acylation of free sterols to steryl esters is catalyzed by two acyltransferases that are localized in the ER [20]. Thus, conceptually sterol esterification in the ER 
membranes could be a means to reduce the "chemical activity" of the lipid in this membrane and thus regulate the equilibration with the plasma membrane pool. The fact that sterol acylation is not essential under standard growth conditions, may indicate that the cell has other means to prevent a potentially harmful accumulation of free sterol in the ER membrane. Steryl esters are re-converted to free sterols through the action of a family of three membrane-anchored lipases that hydrolyze the ester bond to release the free sterol [21]. These lipases essentially complete the sterol esterification and de-esterification cycle and their activities are crucial to regulate cellular levels of free ergosterol under various growth conditions [22].

Saccharomyces cerevisiae does not take up exogenous sterol in the presence of oxygen, but cells become sterol auxotroph in the absence of oxygen or if they are not able to synthesize heme [23]. The molecular basis for the exclusion of sterols under aerobic conditions is not well understood, but it can partially be overcome by expression of a gain-offunction allele of the transcription factor $U P C 2$ or its homologue ECM22, or by overexpression of the transcriptional regulator SUT1 [24,25]. Aerobic uptake of sterols in the UPC2-1 mutant is mediated by upregulation of two ABC transporters, $P D R 11$ and $A U S 1$, and a putative cell wall protein, Dan 1 $[26,27]$. Consistent with a role of these two ABC transporters in sterol uptake, a pdrl $1 \Delta$ aus $1 \Delta$ double mutant does not grow under anaerobic conditions and the double mutant has a greatly reduced rate of steryl ester formation from exogenously supplied sterols $[26,28]$. Consistent with the proposition that the "chemical activity" of the sterol in the plasma membrane is reduced by formation of sterol-sphingolipid complexes, the rate of esterification of exogenously supplied sterols is increased for sterols that have low affinity to form detergent-insoluble domains [28]. In Schizosaccharomyces pombe, on the other hand, lack of oxygen results in the induction of the de novo synthesis of sterols rather than cholesterol auxotrophy as is the case in S. cerevisiae. Sterol biosynthesis in S. pombe is controlled by the regulated proteolysis of membrane bound transcription factors that are orthologous of the mammalian SREBPs (sterol regulatory element binding proteins) [29]. Sterols control the activation of SREPBs by regulating their transport out of the ER and to the Golgi apparatus, where their membrane attachment is cleaved by proteases [4]. Interestingly, the SREBP pathway in $S$. pombe also controls the expression of genes that are important for non-respiratory oxygen consumption and is itself required for anaerobic growth $[29,30]$. Evolution of the SREBPpathway in $S$. pombe thus appears to have taken place at the expense of a sterol uptake pathway.

A defect in sterol uptake and impaired growth under anaerobic conditions was also observed in $S$. cerevisiae cells that lack $A R V I$, a gene that is required for viability of cells that lack steryl esters [31]. ARVI encodes a conserved protein with six putative transmembrane domains and localizes to the ER and Golgi membranes [32]. In addition to their defect in sterol uptake, arvl $\Delta$ mutants have pleiotropic defects in sphingolipid, phospholipid and sterol metabolism, but the precise molecular function of Arv1 remains to be defined [32].

\section{A genetic screen for sterol uptake and transport mutants}

We became interested in sterol trafficking because we isolated mutants in the sterol biosynthetic pathway as being synthetic lethal with mutants in fatty acid elongation. Subsequent work then showed that the combination of defects in certain sterol modifications with defects in sphingolipid synthesis affects the raft association and the stability of integral membrane proteins, such as the proton pumping ATPase [33,34]. These observations together with those made by others (see above) indicate that sterol trafficking is intimately related to sphingolipid metabolism in yeast and mammalian cells.

In an ab initio approach to identify genes that are required for sterol uptake and trafficking in yeast, we screened the yeast deletion mutant collection for genes that are required for growth under anaerobic conditions, i.e. conditions under which yeast is auxotrophic for sterols. Of approximately 37 primary mutants isolated in this screen, 17 affect sterol uptake and/or trafficking as assessed by genetic and biochemical analyses (see Table 1) [35,36]. When incubated with $\left[{ }^{14} \mathrm{C}\right]$-cholesterol, these mutants fail to take up the label and they show no or greatly reduced levels of radiolabeled free and esterified cholesterol. Furthermore, the sterol transport defect in these mutants could be visualized by fluorescent microscopy of cells that had been incubated with NBD-cholesterol, a fluorescent cholesterol derivative, [37]. In wild-type cells, NBD-cholesterol displays intense staining of the plasma membrane and staining of intracellular lipid particles, consistent with our observation that NBD-cholesterol is esterified to steryl esters, which are packaged into intracellular lipid particles [36]. The sterol uptake mutants, on the other hand, show no or only little staining of the plasma membrane and intracellular lipid particles, consistent with a defect in sterol uptake and trafficking (Fig. 1).

Remarkably, the largest class of mutants isolated in this screen is affected in mitochondrial functions. This finding was unexpected and indicates that at least one as yet unidentified mitochondrial function is required for uptake of sterols by yeast under anaerobic conditions. On the other hand, a screen for mutants that affect mitochondrial morphology and inheritance recently revealed that ergosterol biosynthesis is important to maintain mitochondrial morphology even in the presence of oxygen [38]. This observation together with the fact that the ergosterol content of cells drops up to fourfold upon shift to anaerobic conditions could indicate that a reduced sterol content in the mitochondrial membrane could exacerbate an otherwise non-harmful mitochondrial defect, resulting in a complete loss-of-function of that vital organelle and a concomitant defect in sterol uptake [39]. On the other hand, mitochondria themselves are known to be important for sterol metabolism in vertebrate and insect cells. They harbor the cytochrome P450 side chain cleavage enzyme, which converts cholesterol into pregnenolone, the precursor of steroids [40]. Remarkably, heterologous expression of P450 side chain cleavage activity in yeast mitochondria results in 
Table 1

Overview of genes that affect sterol uptake under anaerobic conditions

\begin{tabular}{|c|c|c|c|}
\hline Functional class & Gene & Function & Sterol uptake defect \\
\hline \multirow[t]{2}{*}{ Amino acid metabolism } & HOM6 & Homoserine dehydrogenase; required for biosynthesis of methionine and threonine & Intermediate \\
\hline & TKL1 & $\begin{array}{l}\text { Transketolase I; required for the pentose phosphate pathway and for biosynthesis } \\
\text { of aromatic amino acids }\end{array}$ & Intermediate \\
\hline \multirow{2}{*}{$\begin{array}{l}\text { Regulation of transcription } \\
\text { and translation }\end{array}$} & ROX3 & RNA polymerase II holoenzyme component & Hyperesterification \\
\hline & RPL1B & Structural constituent of the large (60S) ribosomal subunit & Intermediate \\
\hline Vesicular transport & DRS2 & P-type ATPase, potential aminophospholipid translocase & Strong \\
\hline \multirow[t]{2}{*}{ Protein kinases } & BUR2 & Cyclin-dependent protein kinase regulator & Strong \\
\hline & PHO85 & Cyclin-dependent protein kinase, regulates glycogen and phosphate metabolism & Intermediate \\
\hline \multirow[t]{8}{*}{ Mitochondrial function and biogenesis } & ACO1 & Aconitase; required for TCA cycle, mutation leads to glutamate auxotrophy & Intermediate \\
\hline & ATP1 & Alpha-subunit of F1 sector of F1F0 ATP synthase & Strong \\
\hline & ATP2 & Beta-subunit of F1 sector of F1F0 ATP synthase & Intermediate \\
\hline & ATP11 & Molecular chaperone required for assembly of F1 sector of the F1F0 ATP synthase & Intermediate \\
\hline & ATP12 & Molecular chaperone required for assembly of F1 sector of the F1F0 ATP synthase & Intermediate \\
\hline & CAT5 & $\begin{array}{l}\text { May encode a protein involved in one or more mono-oxygenase or hydroxylase } \\
\text { steps of ubiquinone biosynthesis }\end{array}$ & Intermediate \\
\hline & MGM101 & Involved in mitochondrial genome maintenance & Intermediate \\
\hline & UGO1 & Outer membrane protein required for mitochondrial fusion & Intermediate \\
\hline Miscellaneous & GUP1 & $\begin{array}{l}\text { Multimembrane-spanning protein and putative glycerol transporter that is essential } \\
\text { for proton symport of glycerol }\end{array}$ & Strong \\
\hline Unknown function & YJR120 & Hypothetical open reading frame & Strong \\
\hline
\end{tabular}

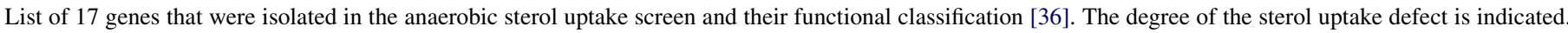

the conversion of endogenously synthesized sterols to pregnenolone, thus indicting that sterols are accessible and thus transported to the $\mathrm{P} 450$ system that is located in the matrix side of the inner mitochondrial membrane [41]. Transport routes for sterols either from the ER or the plasma membrane into mitochondria thus appear to have evolved at some point and may already be present in yeast, where they may also be important for sterol uptake under anaerobic conditions.
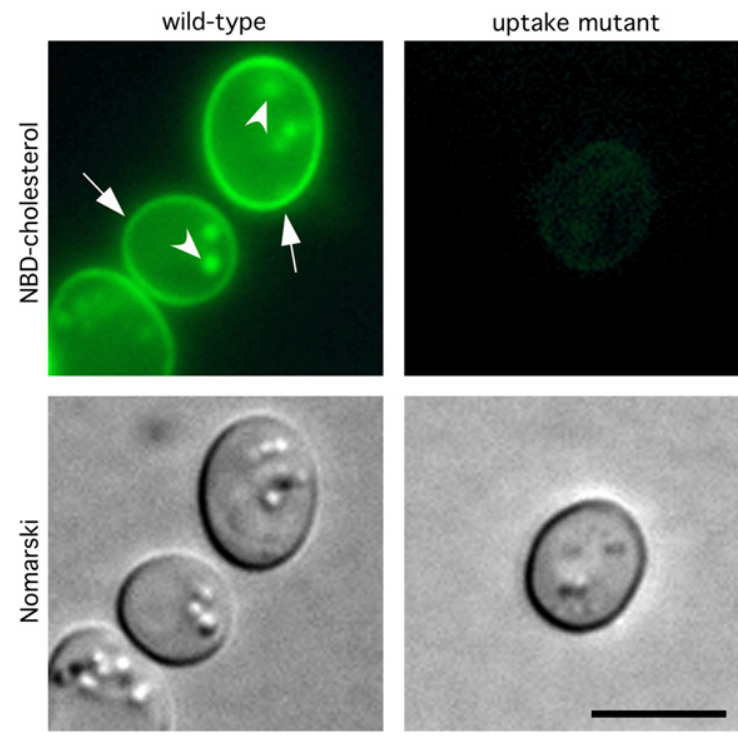

Fig. 1. Subcellular localization of NBD-cholesterol in wild-type and sterol uptake mutants. Heme-deficient wild-type and uptake mutant cells were grown in the presence of NBD-cholesterol for $24 \mathrm{~h}$ at $24^{\circ} \mathrm{C}$ and NBD-cholesterol localization was examined by fluorescent microscopy using identical exposure settings. Plasma membrane and lipid particle staining is indicated by arrows and arrowheads, respectively. Nomarski view of the same visual field is shown below. Bar, $5 \mu \mathrm{m}$.
Taken together, the results of this screen for sterol uptake and transport mutants in yeast revealed 17 genes that are required for growth under anaerobic conditions and for the efficient uptake of cholesterol. Aim of future studies now is to characterize the sterol trafficking defect of these mutants in more detail and in particular to understand the role of mitochondria in sterol uptake. Results of such studies are likely to be relevant for a better understanding of the pathology of human lipid storage diseases and to understand how sterols are partitioned between the different intracellular membranes.

\section{Acknowledgements}

We thank Delphine Micolod for preparation of the figure, Rashi Tiwari for proofreading of this manuscript, and the Swiss National Science Foundation (631-065925) for financial support. We apologize for the primary literature that could not be cited due to space limitations.

\section{References}

[1] T.H. Haines, Do sterols reduce proton and sodium leaks through lipid bilayers? Prog. Lipid Res. 40 (2001) 299-324.

[2] F.R. Maxfield, D. Wustner, Intracellular cholesterol transport, J. Clin. Invest 110 (2002) 891-898.

[3] R.E. Soccio, J.L. Breslow, Intracellular cholesterol transport, Arterioscler. Thromb. Vasc. Biol. 24 (2004) 1150-1160.

[4] J.L. Goldstein, R.A. DeBose-Boyd, M.S. Brown, Protein sensors for membrane sterols, Cell 124 (2006) 35-46.

[5] F.R. Maxfield, I. Tabas, Role of cholesterol and lipid organization in disease, Nature 438 (2005) 612-621.

[6] L.W. Parks, W.M. Casey, Physiological implications of sterol biosynthesis in yeast, Annu. Rev. Microbiol. 49 (1995) 95-116.

[7] N.D. Lees, M. Bard, D.R. Kirsch, Biochemistry and molecular biology of sterol synthesis in Saccharomyces cerevisiae, Crit. Rev. Biochem. Mol. Biol. 34 (1999) 33-47. 
[8] R. Schneiter, B. Brügger, R. Sandhoff, G. Zellnig, A. Leber, M. Lampl, K. Athenstaedt, C. Hrastnik, S. Eder, G. Daum, F. Paltauf, F.T. Wieland, S.D. Kohlwein, Electrospray ionization tandem mass spectrometry (ESIMS/MS) analysis of the lipid molecular species composition of yeast subcellular membranes reveals acyl chain-based sorting/remodeling of distinct molecular species en route to the plasma membrane, J. Cell Biol. 146 (1999) 741-754.

[9] D.P. Sullivan, H. Ohvo-Rekila, N.A. Baumann, C.T. Beh, A.K. Menon, Sterol trafficking between the endoplasmic reticulum and plasma membrane in yeast, Biochem. Soc. Trans. 34 (2006) 356-358.

[10] N.A. Baumann, D.P. Sullivan, H. Ohvo-Rekila, C. Simonot, A. Pottekat, Z. Klaassen, C.T. Beh, A.K. Menon, Transport of newly synthesized sterol to the sterol-enriched plasma membrane occurs via non-vesicular equilibration, Biochemistry 44 (2005) 5816-5826.

[11] A. Radhakrishnan, T.G. Anderson, H.M. McConnell, Condensed complexes, rafts, and the chemical activity of cholesterol in membranes, Proc. Natl. Acad. Sci. USA 97 (2000) 12422-12427.

[12] Y.J. Im, S. Raychaudhuri, W.A. Prinz, J.H. Hurley, Structural mechanism for sterol sensing and transport by OSBP-related proteins, Nature 437 (2005) 154-158.

[13] C.T. Beh, J. Rine, A role for yeast oxysterol-binding protein homologs in endocytosis and in the maintenance of intracellular sterol-lipid distribution, J. Cell Sci. 117 (2004) 2983-2996.

[14] S. Raychaudhuri, Y.J. Im, J.H. Hurley, W.A. Prinz, Non-vesicular sterol movement from plasma membrane to ER requires oxysterol-binding protein-related proteins and phosphoinositides, J. Cell Biol. 173 (2006) 107-119.

[15] S.E. Phillips, P. Vincent, K.E. Rizzieri, G. Schaaf, V.A. Bankaitis, E.A. Gaucher, The diverse biological functions of phosphatidylinositol transfer proteins in eukaryotes, Crit. Rev. Biochem. Mol. Biol. 41 (2006) 21-49.

[16] S.E. Phillips, B. Sha, L. Topalof, Z. Xie, J.G. Alb, V.A. Klenchin, P. Swigart, S. Cockcroft, T.F. Martin, M. Luo, V.A. Bankaitis, Yeast Sec14p deficient in phosphatidylinositol transfer activity is functional in vivo, Mol. Cell 4 (1999) 187-197.

[17] P.Y. Wang, J. Weng, R.G. Anderson, OSBP is a cholesterol-regulated scaffolding protein in control of ERK 1/2 activation, Science 307 (2005) 1472-1476.

[18] P. Wang, Y. Zhang, H. Li, H.K. Chieu, A.L. Munn, H. Yang, AAA ATPases regulate membrane association of yeast oxysterol binding proteins and sterol metabolism, EMBO J. 24 (2005) 2989-2999.

[19] R.B. Bailey, L.W. Parks, Yeast sterol esters and their relationship to the growth of yeast, J. Bacteriol 124 (1975) 606-612.

[20] H. Yang, M. Bard, D.A. Bruner, A. Gleeson, R.J. Deckelbaum, G. Aljinovic, T.M. Pohl, R. Rothstein, S.L. Sturley, Sterol esterification in yeast: a two-gene process, Science 272 (1996) 1353-1356.

[21] R. Köffel, R. Tiwari, L. Falquet, R. Schneiter, The Saccharomyces cerevisiae YLL012/YEH1, YLR020/YEH2, and TGL1 genes encode a novel family of membrane-anchored lipases that are required for steryl ester hydrolysis, Mol. Cell. Biol. 25 (2005) 1655-1668.

[22] R. Köffel, R. Schneiter, Yeh1 constitutes the major steryl ester hydrolase under heme-deficient conditions in Saccharomyces cerevisiae, Euk. Cell 5 (2006) 1018-1025.

[23] R.T. Lorenz, R.J. Rodriguez, T.A. Lewis, L.W. Parks, Characteristics of sterol uptake in Saccharomyces cerevisiae, J. Bacteriol. 167 (1986) 981-985.

[24] S. Bourot, F. Karst, Isolation and characterization of the Saccharomyces cerevisiae SUT1 gene involved in sterol uptake, Gene 165 (1995) 97-102.
[25] K.V. Shianna, W.D. Dotson, S. Tove, L.W. Parks, Identification of a UPC2 homolog in Saccharomyces cerevisiae and its involvement in aerobic sterol uptake, J. Bacteriol. 183 (2001) 830-834.

[26] L.J. Wilcox, D.A. Balderes, B. Wharton, A.H. Tinkelenberg, G. Rao, S.L. Sturley, Transcriptional profiling identifies two members of the ATP-binding cassette transporter superfamily required for sterol uptake in yeast, J. Biol. Chem. 277 (2002) 32466-32472.

[27] P. Alimardani, M. Regnacq, C. Moreau-Vauzelle, T. Ferreira, T. Rossignol, B. Blondin, T. Berges, SUT1-promoted sterol uptake involves the $\mathrm{ABC}$ transporter Aus1 and the mannoprotein Dan1 whose synergistic action is sufficient for this process, Biochem. J. 381 (2004) 195-202.

[28] Y. Li, W.A. Prinz, ATP-binding cassette $(A B C)$ transporters mediate non-vesicular, raft-modulated sterol movement from the plasma membrane to the endoplasmic reticulum, J. Biol. Chem. 279 (2004) 45226-45234.

[29] A.L. Hughes, B.L. Todd, P.J. Espenshade, SREBP pathway responds to sterols and functions as an oxygen sensor in fission yeast, Cell 120 (2005) 831-842.

[30] B.L. Todd, E.V. Stewart, J.S. Burg, A.L. Hughes, P.J. Espenshade, Sterol regulatory element binding protein is a principal regulator of anaerobic gene expression in fission yeast, Mol. Cell. Biol. 26 (2006) 2817-2831.

[31] A.H. Tinkelenberg, Y. Liu, F. Alcantara, S. Khan, Z. Guo, M. Bard, S.L. Sturley, Mutations in yeast ARV1 alter intracellular sterol distribution and are complemented by human ARV1, J. Biol. Chem. 275 (2000) 40667-40670.

[32] E. Swain, J. Stukey, V. McDonough, M. Germann, Y. Liu, S.L. Sturley, J.T. Nickels Jr., Yeast cells lacking the ARV1 gene harbor defects in sphingolipid metabolism. Complementation by human ARV1, J. Biol. Chem. 277 (2002) 36152-36160.

[33] M. Eisenkolb, C. Zenzmaier, E. Leitner, R. Schneiter, A specific structural requirement for ergosterol in long-chain fatty acid synthesis mutants important for maintaining raft domains in yeast, Mol. Biol. Cell 13 (2002) 4414-4428.

[34] B. Gaigg, B. Timischl, L. Corbino, R. Schneiter, Synthesis of sphingolipids with very long chain fatty acids but not ergosterol is required for routing of newly synthesized plasma membrane ATPase to the cell surface of yeast, J. Biol. Chem. 280 (2005) 22515-22522.

[35] S. Reiner, D. Micolod, R. Schneiter, Saccharomyces cerevisiae, a model to study sterol uptake and transport in eukaryotes, Biochem. Soc. Trans. 33 (2005) 1186-1188.

[36] S. Reiner, D. Micolod, G. Zellnig, R. Schneiter, A genome wide screen reveals a role of mitochondria in anaerobic uptake of sterols in yeast, Mol. Biol. Cell 17 (2006) 90-103.

[37] C.P. Sparrow, S. Patel, J. Baffic, Y.S. Chao, M. Hernandez, M.H. Lam, J. Montenegro, S.D. Wright, P.A. Detmers, A fluorescent cholesterol analog traces cholesterol absorption in hamsters and is esterified in vivo and in vitro, J. Lipid Res. 40 (1999) 1747-1757.

[38] K. Altmann, B. Westermann, Role of essential genes in mitochondrial morphogenesis in Saccharomyces cerevisiae, Mol. Biol. Cell 16 (2005) 5410-5417.

[39] F. Paltauf, G. Schatz, Promitochondria of anaerobicallly grown yeast. II. Lipid composition, Biochemistry 8 (1969) 335-339.

[40] W.L. Miller, Mitochondrial specificity of the early steps in steroidogenesis, J. Steroid Biochem. Mol. Biol. 55 (1995) 607-616.

[41] C. Duport, R. Spagnoli, E. Degryse, D. Pompon, Self-sufficient biosynthesis of pregnenolone and progesterone in engineered yeast, Nat. Biotechnol 16 (1998) 186-189. 